\title{
Association of serum brain-derived neurotrophic factor (BDNF) and tumor necrosis factor-alpha (TNF- $\alpha$ ) with diagnosis of delirium in oncology inpatients
}

\author{
Cristiano Brum, ${ }^{1,2}$ Laura Stertz, ${ }^{3}$ Ericksen Borba, ${ }^{4}$ Danielle Rumi, ${ }^{5}$ Flávio Kapczinski, ${ }^{3}$ \\ Analuiza Camozzato ${ }^{1,2}$ \\ ${ }^{1}$ Graduate Program in Health Sciences, Universidade Federal de Ciências da Saúde de Porto Alegre (UFCSPA), Porto Alegre, RS, Brazil. \\ ${ }^{2}$ Hospital Santa Rita, Irmandade Santa Casa de Misericórdia de Porto Alegre (ISCMPA), Porto Alegre, RS, Brazil. ${ }^{3}$ Molecular Psychiatry \\ Laboratory, National Science and Technology Institute for Translational Medicine (INCT-TM), Hospital de Clínicas de Porto Alegre (HCPA), \\ Universidade Federal do Rio Grande do Sul (UFRGS), Porto Alegre, RS, Brazil. ${ }^{4}$ Graduate Program in Medical Sciences, UFRGS, Porto \\ Alegre, RS, Brazil. ${ }^{5}$ School of Medicine, UFCSPA, Porto Alegre, RS, Brazil.
}

\begin{abstract}
Objective: To evaluate brain-derived neurotrophic factor (BDNF) and tumor necrosis factor- $\alpha$ (TNF- $\alpha)$ blood levels as disease biomarkers of delirium in oncology inpatients.

Methods: Seventeen oncology inpatients with delirium, 28 oncology inpatients without delirium, and 25 non-oncology controls (caregivers) were consecutively recruited from a Brazilian cancer center. This sample was matched by age, sex, and education level. The Confusion Assessment Method, the Mini-Mental State Examination, and the Digit Span Test were administered to ascertain delirium diagnosis. BDNF and TNF- $\alpha$ levels were measured by the Sandwich-ELISA method and flow cytometry, respectively. Blood samples were collected immediately after clinical evaluation.

Results: Oncology inpatients (with and without delirium) showed significantly lower BDNF levels compared with non-oncology controls $(F=13.830 ; p=0.001)$. TNF- $\alpha$ levels did not differ between the three groups.

Conclusion: A cross-sectional relationship of BDNF and TNF- $\alpha$ blood levels with delirium in oncology inpatients was not demonstrated. The association between cancer and reduced serum BDNF levels may be mediated by confounding factors.
\end{abstract}

Keywords: Cancer; delirium; tumor necrosis factor- $\alpha$; brain-derived neurotrophic factor

\section{Introduction}

Delirium is one of the most frequent neuropsychiatric complications in oncology patients. Its incidence rate ranges from 16 to $85 \%$ during hospital admission depending on the stage of the underlying disease. ${ }^{1}$ Delirium is a predictor of morbidity and mortality that frequently goes under-recognized in clinical practice. ${ }^{1}$ In addition, its pathophysiology is still relatively unknown. ${ }^{2}$ The identification of biomarkers for delirium may contribute to the diagnosis of delirium and to a better understanding of its pathophysiology.

Some authors have described the role of systemic inflammation. According to the literature, systemic inflammation starts with the activation of central nervous system parenchymal cells. Next, there is expression of proinflammatory cytokines and inflammatory mediators in the brain. Finally, there is neuronal and synaptic dysfunction leading to delirium. ${ }^{2-6}$ The high prevalence of delirium in sepsis, ${ }^{7}$ surgery, ${ }^{8,9}$ and treatment of cancer with interleukin and

Correspondence: Analuiza Camozzato, Av. Taquara, 564/306, CEP 90460-210, Porto Alegre, RS, Brazil.

E-mail: anacamoz@gmail.com

Submitted May 19 2014, accepted Oct 202014. interferon ${ }^{10,11}$ and the high levels of inflammatory markers observed in many etiologies of delirium ${ }^{8,9,12,13}$ support this theory.

Tumor necrosis factor-alpha (TNF- $\alpha$ ) is one of the proinflammatory cytokines responsible for inflammation, immunity, and cellular homeostasis. TNF- $\alpha$ also plays a critical role in tumor proliferation, migration, invasion, and angiogenesis. In addition, TNF- $\alpha$ is a key regulator of the tumor microenvironment. ${ }^{14,15}$ This cytokine could also be linked to the pathophysiology of delirium. ${ }^{4,5}$ TNF- $\alpha$ may mediate excitotoxic neurodegeneration by inhibiting the activity of the insulin-like growth factor I (IGF-I), a neuroprotective peptide that stresses the interaction between neurotrophic and inflammatory factors. ${ }^{16}$ Previous synaptic loss and microglial priming caused by aging and neurodegeneration are predisposing factors associated with acute cognitive impairment in patients with delirium. ${ }^{3}$ Peripheral TNF- $\alpha$ induced by a systemic inflammatory process also plays an important role in microglial activation and further cytokine release in the cerebral tissue, which has a deleterious effect on neuronal functions. ${ }^{4}$

The brain-derived neurotrophic factor (BDNF), a neurotrophin found in abundance in the hippocampus and cerebral cortex, ${ }^{17}$ has also been considered to play a role 
in the pathophysiology of delirium. ${ }^{7}$ The effect of BDNF on synaptic plasticity, dendritic growth, normal maturation of neural development pathways, ${ }^{18}$ and long-term memory consolidation ${ }^{19,20}$ supports its possible contribution to delirium. In addition, increased oxidative stress, which has been suggested as one of the processes leading to the development of delirium, ${ }^{21}$ has been associated with decreased BDNF levels. ${ }^{22}$ Furthermore, the age-related reductions in serum levels of $\mathrm{BDNF}^{23,24}$ and its association with the decrease in volume of the hippocampus and in memory performance ${ }^{25}$ may be related to the vulnerability of the elderly to develop delirium. ${ }^{1,3}$ High levels of BDNF and its subsequent reduction were found to be predictive of delirium in intensive care unit patients. ${ }^{26}$

This study aimed to evaluate the role of BDNF and TNF- $\alpha$ serum levels as disease markers of delirium in oncology inpatients. Considering the theory of increased oxidative stress linked to delirium, ${ }^{21}$ the authors' hypothesis was that BDNF levels would be reduced during the phenomenological presentation of this acute neuropsychiatric condition. It was also hypothesized that TNF- $\alpha$ levels would be elevated during the clinical presentation of this syndrome as an expression of a potentially reversible inflammatory brain disease.

\section{Methods}

\section{Study design}

We conducted a case-control study comparing three different groups matched according to sex, age, and education. The first group was composed of oncology inpatients with delirium, the second group enrolled oncology inpatients without delirium, and the third group was comprised of non-oncology subjects.

\section{Participants}

Oncology inpatients (with and without delirium) were consecutively recruited from the Cancer Center at Hospital Santa Casa, Porto Alegre, Brazil, from January to August 2011. Non-oncology subjects were selected among cancer inpatients' caregivers during hospitalization. The inclusion criteria for the groups with cancer were diagnosis of breast, head and neck, lung, bladder, uterine, gastric, and colon cancers. The presence of a proxy during evaluation was required in cases of delirium. Exclusion criteria were aphasia; severe hearing and visual impairments, because they complicate the patients' assessment; prior history of symptoms of dementia; presence of diagnostic ovarian cancer, myelomas, prostate and lymphoid tumors, medullary thyroid carcinoma, and neuroblastoma to avoid possible confounder related to BDNF and tumorogenesis ${ }^{27-29}$; and presence of any psychiatric disorder in oncology inpatients without delirium and non-oncology controls. Ten patients with major depression, seven patients with prostate cancer, four patients with ovarian cancer, and two patients with previous dementia were excluded. Figure 1 shows the recruitment process flowchart.

\section{Assessments}

The oncology staff of the Hospital Santa Casa was informed about the protocol and authorized the assessment of their patients. The diagnosis of cancer was established based on clinical history and confirmed by an anatomical pathology study. Demographic data were also obtained.

Before the beginning of the study, the nursing staff of the hospital was trained by the research team to improve delirium recognition. All suspected cases of delirium identified by nurses were further evaluated by a consultant

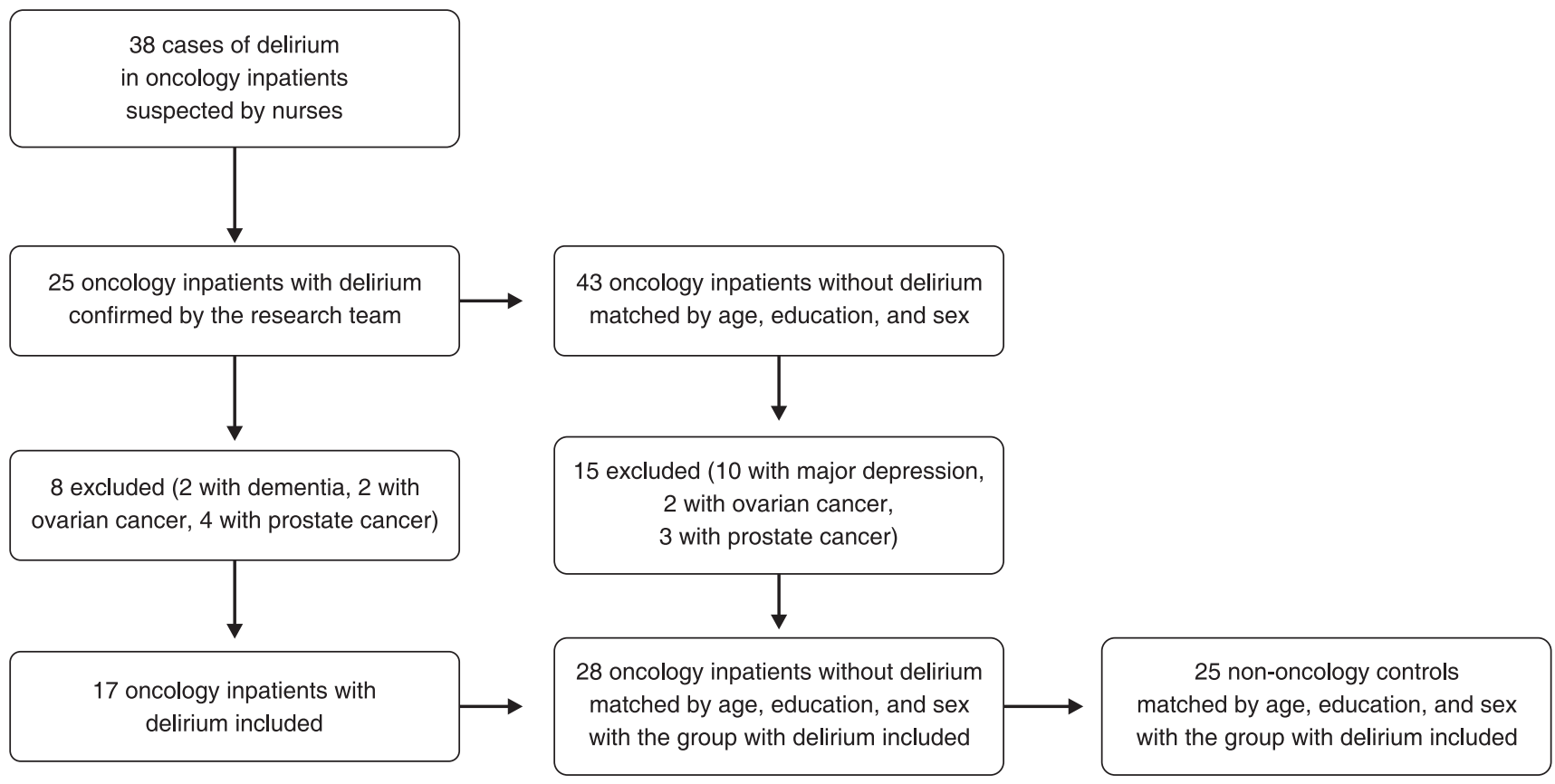

Figure 1 Recruitment process flowchart. 
psychiatrist or three previously trained members of the research team in order to confirm the diagnosis. Clinical history was obtained from patients and informants. The Confusion Assessment Method (CAM), ${ }^{30,31}$ the MiniMental State Examination (MMSE), and the Digit Span Test were administered to all oncology inpatients in order to evaluate the presence of delirium. A diagnosis of delirium by the CAM requires the presence of acute onset and fluctuating course, inattention, and either disorganized thinking or altered level of consciousness. The CAM is scored based on an interview with the patient including at least a brief cognitive assessment. The MMSE and the Digit Span Test were thus used as brief cognitive measures to confirm the diagnosis of delirium suggested by the CAM. ${ }^{30}$ The MMSE and the Digit Span Test were also administered to non-oncology subjects. The Brazilian version of the MINI International Neuropsychiatric Interview ${ }^{32}$ was used to identify any psychiatric disorders in oncology inpatients without delirium and non-oncology controls. Those subjects who presented any psychiatric disorders were not included in the study. A comprehensive clinical history was obtained to exclude significant clinical diseases in the non-oncology controls. For each oncology inpatient with delirium included in the study, the team searched for an oncology inpatient without delirium and a healthy control matched according to age, education, and sex.

Immediately after the clinical and psychiatric assessment, blood was collected from all participants by a senior nurse without anticoagulant. Within 3 hours, serum was separated by centrifugation at $3000 \times \mathrm{G}$ at room temperature. The supernatant was stored at $-80^{\circ} \mathrm{C}$ until the assay. BDNF serum levels were determined by sandwich-ELISA method using BDNF monoclonal antibodies (R\&D Systems, Minneapolis, MN, USA) according to the manufacturer's instructions. The limit of detection for BDNF was $7.8 \mathrm{pg} / \mathrm{mL}$ and the range of detection was from $7.8 \mathrm{pg} / \mathrm{mL}$ to $500 \mathrm{pg} / \mathrm{mL}$. Also following the manufacturer's instructions, TNF- $\alpha$ was measured using fluorescence detection by flow cytometry. The device used was the BD $^{\text {TM }}$ CBA Human TNF Enhanced Sensitivity Flex (BD Biosciences, San Diego, CA, USA). The theoretical limit of detection of TNF- $\alpha$ is $67.3 \mathrm{fg} / \mathrm{mL}$ and it was determined by evaluating the estimated result of the median fluorescence intensity (MFI) of the negative control $(0 \mathrm{fg} / \mathrm{mL}, \mathrm{n}=30)+2$ standard deviations. The laboratory assessment was blinded to the clinical status of the subjects.

All participants and their proxies (in cases of delirium) were fully informed about the study and signed a written consent form. The study was approved by the Ethics
Committee of the Universidade Federal de Ciências da Saúde de Porto Alegre, Porto Alegre, Brazil (protocol number 979/09). All procedures are in accordance with the Code of Ethics of the World Medical Association (Declaration of Helsinki) for experiments involving humans.

\section{Statistical analysis}

The Shapiro-Wilk test was used to test the normality of all the variables studied. Descriptive analyses of demographic and clinical data were also performed. Analysis of variance with Tukey's post-hoc comparison was used to identify between-group differences in terms of BDNF and TNF- $\alpha$ serum levels because the distribution of these variables was parametric. The same analysis was used to compare age and educational level between the groups. The chi-square test was used to compare sex between the groups. The effect of sex and chemotherapy on BDNF and TNF- $\alpha$ serum levels was analyzed by Student's $t$ test. The Pearson correlation test was used to evaluate the correlation of BDNF and TNF- $\alpha$ serum levels with educational level and age. A threshold $p$-value was set at 0.05 to be statistically significant. All statistical analyses were carried out using the SPPS version 18.0.

The statistical power of the sample size was calculated after data analysis based on the mean differences observed in the study and $95 \%$ confidence level using the OpenEpi version 2, open source calculator (Power, http://www. openepi.com/Power/PowerMean.htm).

\section{Results}

A sample of 17 oncology inpatients with delirium, 28 oncology inpatients without delirium, and 25 non-oncology controls was evaluated. The mean age of the whole sample was 65.19 (SD 8.29), ranging from 41 to 89 years. The comparative analysis of demographic data showed no difference between the three groups (Table 1).

The most frequent cancer diagnosis was breast (13\%) and lung cancer (13\%), followed by colon $(10 \%)$ and stomach $(6 \%)$ cancer. Five (29\%) oncology inpatients with delirium and seven $(25 \%)$ oncology inpatients without delirium were using antibiotics. Approximately half of the total oncology sample had metastasis and was undergoing chemotherapy. Oncology inpatients with delirium did not have a significantly higher rate of metastasis $(44.4 \%)$ than oncology inpatients without delirium $(55.6 \%)(p=0.114)$ (chi-square association test). Forty percent of oncology

Table 1 Comparative analyses of demographic data between the three groups

\begin{tabular}{lcccc}
\hline Variable & $\begin{array}{c}\text { Oncology inpatients with } \\
\text { delirium }(\mathrm{n}=17)\end{array}$ & $\begin{array}{c}\text { Oncology inpatients without } \\
\text { delirium }(\mathrm{n}=28)\end{array}$ & $\begin{array}{c}\text { Non-oncology healthy } \\
\text { controls }(\mathrm{n}=25)\end{array}$ & $\mathrm{p}$-value \\
\hline Age (years)* & $68.65 \pm 10.70$ & $62.81 \pm 4.85$ & $65.40 \pm 8.85$ & 0.07 \\
Education (years)* $^{5.38 \pm 2.70}$ & & $7.19 \pm 3.07$ & $8.96 \pm 6.48$ & \\
Sex $^{\dagger}$ & $7(22)$ & & & \\
Female & $10(26)$ & $15(47)$ & $10(31)$ & 0.08 \\
Male & $13(34)$ & $15(40)$ & \\
\hline
\end{tabular}

Data presented as mean \pm standard deviation or $n(\%)$.

* One-way analysis of variance (ANOVA): $\mathrm{F}=2.723$ for age; $\mathrm{F}=2.612$ for education.

Chi-square test: $\chi^{2}=1.167$. 
Table 2 Comparison of BDNF and TNF- $\alpha$ levels between oncology inpatients with delirium, oncology inpatients without delirium, and non-oncology healthy subjects (one-way ANOVA)

\begin{tabular}{lcccc}
\hline Variable & $\begin{array}{c}\text { Oncology inpatients with } \\
\text { delirium }(n=17)\end{array}$ & $\begin{array}{c}\text { Oncology inpatients without } \\
\text { delirium }(n=28)\end{array}$ & $\begin{array}{c}\text { Non-oncology healthy } \\
\text { controls }(n=25)\end{array}$ & $p$-value \\
\hline BDNF pg/mL $*^{\dagger}$ & $17.47 \pm 11.76^{\mathrm{a}}$ & $22.98 \pm 12.85^{\mathrm{b}}$ & $36.55 \pm 11.57^{\mathrm{c}}$ & $0.001^{*}$ \\
TNF- $\alpha \mathrm{pg} / \mathrm{mL}^{\dagger}$ & $0.350 \pm 0.147$ & $0.368 \pm 0.170$ & $0.328 \pm 0.206$ & 0.749 \\
\hline
\end{tabular}

Data presented as mean \pm standard deviation.

ANOVA $=$ analysis of variance; BDNF $=$ brain-derived neurotrophic factor; TNF- $\alpha=$ tumor necrosis factor-alpha.

* Tukey's post-hoc comparison a, b < c.

$\dagger$ One-way ANOVA: $F=13.830$ for BDNF; $F=0.290$ for TNF- $\alpha$ levels.

inpatients with delirium were on chemotherapy compared with $60 \%$ of oncology inpatients without delirium $(p=$ 0.656) (chi-square association test). Fifty-six percent of oncology inpatients with delirium and $55.5 \%$ of those oncology inpatients without delirium were using benzodiazepines. The frequency of opioid use was $58 \%$ for oncology inpatients with delirium and $63 \%$ for oncology inpatients without delirium.

BDNF serum levels were not significantly correlated with age and education $(r=0.22, p=0.08$ and $r=0.23, p=0.07$, respectively). TNF- $\alpha$ serum levels showed a weak positive significant correlation $(r=0.3, p=0.05)$ with educational level, but not with age. BDNF and TNF- $\alpha$ levels did not differ significantly between patients on current chemotherapy $(t=-0.78, \mathrm{p}=0.43 ; t=-0.21, \mathrm{p}=0.83)$ and between $\operatorname{sex}(t=-1.70, \mathrm{p}=0.09 ; t=-0.66, \mathrm{p}=0.51)$.

Oncology inpatients (with and without delirium) showed significantly lower BDNF levels in comparison with those found in the control group of non-oncology controls ( $F=$ 13.830; $p=0.001$ ) (Table 2). Although without statistical significance, there was a decreasing gradient from oncology inpatients with delirium to non-oncology controls. This result was illustrated in a box plot graph (Figure 2). TNF- $\alpha$ levels did not differ between the three groups (Table 2).

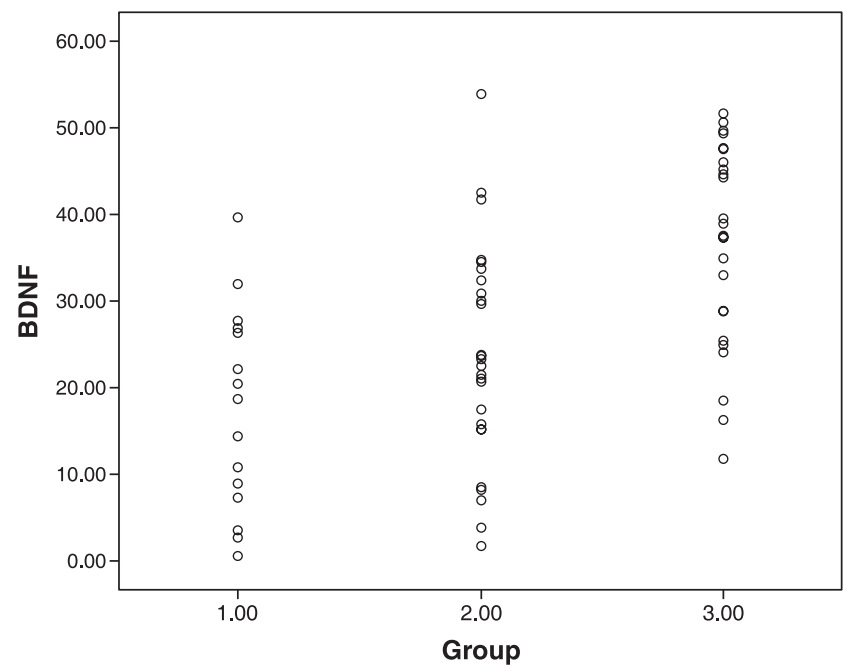

Figure 2 Brain-derived neurotrophic factor (BDNF) levels between oncology inpatients with and without delirium and non-oncology healthy subjects (scatter plot graph). Group 1 = oncology inpatients with delirium; Group 2 = oncology inpatients without delirium; Group 3 = non-oncology healthy controls.
The power of mean differences of BDNF levels between oncology inpatients with delirium and non-oncology subjects was $99.94 \%$ and $98.15 \%$ between oncology inpatients without delirium and non-oncology subjects.

\section{Discussion}

This study demonstrated that oncology inpatients (with and without delirium) showed significantly lower BDNF serum levels than non-oncology controls. The lowest BDNF serum levels were found in oncology inpatients with delirium. Nevertheless, their levels were statistically similar to those of oncology inpatients without delirium. TNF- $\alpha$ serum levels showed no difference between the three groups, despite previous hypotheses that TNF- $\alpha$ serum levels would be increased in patients with delirium compared with those without delirium.

Our initial hypothesis that BDNF serum levels would be significantly lower during the delirium phenomenological presentation in oncology inpatients with delirium in comparison with those without delirium and non-oncology controls has not been confirmed. Because blood was collected while our patients were displaying the clinical presentation of delirium, we did not find a cross-sectional relationship between delirium and BDNF levels in oncology inpatients. We have chosen a sample of oncology inpatients because of their high risk of delirium. ${ }^{1}$ Even those patients who did not have delirium signs at that moment blood was collected share a similar risk. Both groups had low BDNF levels in comparison with healthy controls. Only a longitudinal study design, including blood collection at hospital admission and a daily follow-up of the patients to detect delirium, would make it possible to test the hypothesis that BDNF levels may be a risk factor for delirium incidence in oncology patients. The role of BDNF as a risk factor for this condition was already demonstrated in intensive care unit (ICU) patients. ${ }^{26}$ Furthermore, lower BDNF plasma levels were independently associated with mortality, even in the absence of clinically detectable brain dysfunction in ICU patients. ${ }^{33}$ Additionally, for those oncology patients who were hospitalized, the mortality risk is well-known. ${ }^{34}$

The current results may suggest a relationship between having cancer and having reduced serum levels of BDNF, although this association may be found due to potential confounding factors. The biology of the different types of cancer found in the patients enrolled in the study was diverse and might have impacted the result. Furthermore, the elevated use of benzodiazepine among the sample could be another factor related to low BDNF levels. ${ }^{35}$ 
Also, we did not measure other potential confounding factors for low BDNF levels in the oncology sample, such as body mass index, minor depressive symptoms, or minor clinical diseases or presence of hypoxemia. Conversely, it is reasonable to assume that having cancer is stressful, and oncology inpatients probably face chronic stress that persists for hours, days, weeks, and even months. An experimental model showed that acute stress induced a significant increase in BDNF mRNA and protein, while repeated chronic stress resulted in their reduction. ${ }^{34}$ Therefore, we can hypothesize that chronic stress could be induced by cancer which, in turn, induced a reduction of BDNF.

Furthermore, the small sample size is another factor that could explain the non-significance of BDNF levels between the oncology inpatients with delirium and those oncology inpatients without this syndrome. However, a post-hoc analysis corroborated the statistical power of our sample. Additionally, we did not measure other substances related to the pathophysiology of delirium, such as IG-F1, IL-8, IL6 , cortisol, C-reactive protein, and S-100 $\beta$, as well as ApoE 4 allele ${ }^{36}$ which could have interacted with BDNF levels in order to express the symptoms of delirium.

TNF- $\alpha$ serum level was similar in the three groups. According to the delirium inflammatory hypotheses, ${ }^{2-6}$ which emphasize the role of stress-induced cytokines, we expected increased levels of this pro-inflammatory cytokine in oncology inpatients with delirium. In addition, the role of this cytokine as one of the major mediators of cancerrelated inflammation ${ }^{15}$ strengthened this theory. Despite the theoretical potential role of TNF- $\alpha$ in delirium associated with cancer, abnormalities in this cytokine have not been found in other conditions associated with delirium. ${ }^{12,37}$ Conversely, our results could be explained by an ephemeral increase in TNF- $\alpha$ in the blood, which could not exactly reflect its central nervous system levels. Increased TNF- $\alpha$ could last a few hours in the blood and this transitory rise would be sufficient to activate the neuroinflammatory cascade. An experimental study reported that after injections of single systemic lipopolysaccharide (LPS, $5 \mathrm{mg} / \mathrm{kg}$, i.p.) in wild mice, peripheral TNF- $\alpha$ was detectable in the serum for 9 hours, in the liver for 1 week, while it remained at a high level in the brain for 10 months. ${ }^{38}$

Another explanation for this finding was the myriad of causes of delirium in oncology inpatients making the identification of the main precipitant factor very difficult. The multiple precipitant factors could have a different correlation with TNF- $\alpha$ levels. Furthermore, it was not possible to obtain the TMN staging for all oncology inpatients; therefore, the potential effect of this variable on BDNF and TNF- $\alpha$ levels was not studied. This issue could be considered in future research.

Small sample size, heterogeneity of cancer types, and lack of control of the many confounding factors described above were limitations of the current study. The multifactorial causes of delirium and the clinical complexity of oncology patients may interfere with the study of this heterogeneous population. These aspects may also have an impact on the results because many potential factors related to delirium may be underestimated and/or unrecognized.
Nonetheless, the high prevalence of delirium in this population challenges new research on diagnostic and preventive strategies. One of the strengths of our study was to evaluate this specific group. In spite of the vast literature about the role of BDNF in many neuropsychiatric disorders, its role in delirium has been the subject of more theoretical discussions than clinical investigations. Longitudinal studies with larger sample size of oncology inpatients could better evaluate the role of BDNF in delirium in this population.

\section{Disclosure}

The authors report no conflicts of interest.

\section{References}

1 Caraceni A, Simonetti F. Palliating delirium in patients with cancer. Lancet Oncol. 2009;10:164-72.

2 MacLullich AM, Ferguson KJ, Miller T, de Rooij SE, Cunningham C. Unravelling the pathophysiology of delirium: a focus on the role of aberrant stress responses. J Psychosom Res. 2008;65:229-38.

3 Murray C, Sanderson DJ, Barkus C, Deacon RM, Rawlins JN, Bannerman DM, et al. Systemic inflammation induces acute working memory deficits in the primed brain: relevance for delirium. Neurobiol Aging. 2010;33:603-16.

4 van Gool WA, van de Beek D, Eikelenboom P. Systemic infection and delirium: when cytokines and acetylcholine collide. Lancet. 2010;375:773-5.

5 Cerejeira J, Firmino H, Vaz-Serra A, Mukaetova-Ladinska EB. The neuroinflammatory hypothesis of delirium. Acta Neuropathol. 2010;119:737-54

6 Simone MJ, Tan ZS. The role of inflammation in the pathogenesis of delirium and dementia in older adults: a review. CNS Neurosci Ther. 2011;17:506-13.

7 Gunther ML, Morandi A, Ely EW. Pathophysiology of delirium in the intensive care unit. Crit Care Clin. 2008;24:45-65.

8 Rudolph JL, Ramlawi B, Kuchel GA, McElhaney JE, Xie D, Sellke FW, et al. Chemokines are associated with delirium after cardiac surgery. J Gerontol A Biol Sci Med Sci. 2008;63:184-9.

9 van Munster BC, Korevaar JC, Zwinderman AH, Levi M, Wiersinga WJ, De Rooij SE. Time-course of cytokines during delirium in elderly patients with hip fractures. J Am Geriatr Soc. 2008;56:170409.

10 Rosenberg S, Loetz M, Yang J, Aebersold PM, Linehan WM, Seipp CA, et al. Experience with the use of high-dose interleukin-2 in the treatment of 652 cancer patients. Ann Surg. 1989;210:47484.

11 van Steijn JH, Nieboer P, Hospers GA, de Vries EG, Mulder NH. Delirium after interleukin-2 and alpha-interferon therapy for renal cell carcinoma. Anticancer Res. 2001;21:3699-700.

12 Adamis D, Lunn M, Martin FC, Treloar A, Gregson N, Hamilton G, et al. Cytokines and IGF-I in delirious and non-delirious acutely ill older medical inpatients. Age Ageing. 2009;38:326-32.

13 MacLullich AM, Edelshain BT, Hall RJ, de Vries A, Howie SE, Pearson A, et al. Cerebrospinal fluid interleukin-8 levels are higher in people with hip fracture with perioperative delirium than in controls. J Am Geriatr Soc. 2011;59:1151-3.

14 Balkwill F. Tumour necrosis factor and cancer. Nat Rev Cancer. 2009;361-71.

$15 \mathrm{Wu}$ Y, Zhou BP. TNF- $\alpha / \mathrm{NF}-\kappa \mathrm{B} / \mathrm{S}$ nail pathway in cancer cell migration and invasion. Br J Cancer. 2010;102:639-44.

16 Venters HD, Tang Q, Liu Q, VanHoy RW, Dantzer R, Kelley KW. A new mechanism of neurodegeneration: a proinflammatory cytokine inhibits receptor signaling by a survival peptide. Proc Natl Acad Sci U S A. 1999;96:9879-84.

17 Ernfors $\mathrm{P}$, Wetmore $\mathrm{C}$, Olson L, Persson $\mathrm{H}$. Identification of cells in rat brain and peripheral tissues expressing mRNA for members of the nerve growth factor family. Neuron. 1990;5:511-26. 
18 Poo MM. Neurotrophins as synaptic modulators. Nat Rev Neurosci. 2001;2:24-32.

19 Alonso M, Vianna MR, Depino AM, Mello e Souza T, Pereira P, Szapiro $\mathrm{G}$, et al. BDNF-triggered events in the rat hippocampus are required for both short- and long-term memory formation. Hippocampus. 2002; 12:551-60.

20 Pang PT, Lu B. Regulation of late-phase LTP and long-term memory in normal and aging hippocampus: role of secreted proteins IPA and BDNF. Ageing Res Rev. 2004;3:407-30.

21 Maldonado JR. Neuropathogenesis of delirium: review of current etiologic theories and common pathways. Am J Geriatr Psychiatry. 2013;21:1190-222.

22 Kapcinski F, Frey BN, Andreazza AC, Kauer-Sant'Anna M, Cunha AB, Post RM. Increased oxidative stress as a mechanism for decreased BDNF levels in acute manic episodes. Rev Bras Psiquiatr. 2008;30: 243-5.

23 Lommatzsch M, Zingler D, Schuhbaeck K, Schloetcke K, Zingler C, Schuff- Werner $\mathrm{P}$, et al. The impact of age, weight and gender on BDNF levels in human platelets and plasma. Neurobiol Aging. 2005;26:115-23.

24 Ziegenhorn AA, Schulte-Herbrüggen O, Danker-Hopfe H, Malbranc $M$, Hartung HD, Anders D, et al. Serum neurotrophins: a study on the time course and influencing factors in a large old age sample. Neurobiol Aging. 2007;28:1436-45.

25 Erickson KI, Prakash RS, Voss MW, Chaddock L, Heo S, McLaren $\mathrm{M}$, et al. Brain-derived neurotrophic factor is associated with agerelated decline in hippocampal volume. J Neurosci. 2010;30:536875.

26 Grandi C, Tomasi CD, Fernandes K, Stertz L, Kapczinski F, Quevedo J, et al. Brain-derived neurotrophic factor and neuron-specific enolase, but not $S 100 \beta$, levels are associated to the occurrence of delirium in intensive care unit patients. J Crit Care. 2011:26;133-7.

27 Bronzetti E, Artico M, Forte F, Pagliarella G, Felici LM, D’Ambrosio $\mathrm{A}$, et al. A possible role of BDNF in prostate cancer detection. Oncol Rep. 2008;19:969-74.
28 Thiele CJ, Li Z, McKee AE. On Trk-the TrkB signal transduction pathway is an increasingly important target in cancer biology. Clin Cancer Res. 2009;5:5962-7.

29 Au CW, Siu MK, Liao X, Wong ES, Ngan HY, Tam KF, et al. Tyrosine kinase $B$ receptor and BDNF expression in ovarian cancers - effect on cell migration, angiogenesis and clinical outcome. Cancer Lett. 2009; 281:151-61.

30 Inouye SK, Van Dyck CH, Alessi CA, Balkin S, Siegal AP, Horwitz RI. Clarifying confusion: the confusion assessment method. A new method for detection of delirium. Ann Intern Med. 1990;113:941-8.

31 Fabbri RM, Moreira MA, Garrido R, Almeida OP. Validity and reliability of the Portuguese version of the Confusion Assessment Method (CAM) for the detection of delirium in the elderly. Arq Neuropsiquiatr. 2001;59:175-9.

32 Amorim P. Mini International Neuropsychiatric Interview (MINI): validação de entrevista breve para diagnóstico de transtornos mentais. Rev Bras Psiquiatr. 2000;22:106-15.

33 Ritter C, Miranda AS, Giombelli VR, Tomasi CD, Comim CM, Teixeira AL, et al. Brain-derived neurotrophic factor plasma levels are associated with mortality in critically ill patients even in the absence of brain injury. Crit Care. 2012;16:R234.

34 Rocque GB, Barnett AE, Illig LC, Eickhoff JC, Bailey HH, Campbell TC, et al. Inpatient hospitalization of oncology patients: are we missing an opportunity for end-of-life care? J Oncol Pract. 2013;9:51-4.

35 Cao L, During MJ. What is the brain-cancer connection? Annu Rev Neurosci. 2012;35:331-45.

36 Khan BA, Zawahiri M, Campbell NL, Boustani MA. Biomarkers for delirium-a review. J Am Geriatr Soc. 2011;59:S256-61.

37 Plaschke K, Fichtenkamm P, Schramm C, Hauth S, Martin E, Verch $M$, et al. Early postoperative delirium after open-heart cardiac surgery is associated with decreased bispectral EEG and increased cortisol and interleukin-6. Intensive Care Med. 2010;36:2081-9.

38 Qin L, Wu X, Block ML, Liu Y, Breese GR, Hong JS, et al. Systemic LPS causes chronic neuroinflammation and progressive neurodegeneration. Glia. 2007;55:453-62. 